Recepción: 04 / 05/ 2018

Aceptación: 15 / 06 / 2018

Publicación: 01 / 08 / 2018

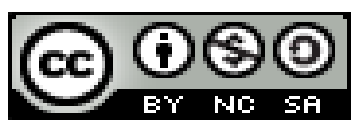

Ciencias Ambientales

Artículo Científico

\title{
Prevalencia de Toxocara canis y su incidencia zoonótica ambiental en niños de la ciudad de Jipijapa
}
Prevalence of Toxocara canis and its environmental zoonotic incidence in children of the city of Jipijapa

Prevalência de Toxocara canis e sua incidência zoonótica ambiental em crianças da cidade de Jipijapa

Néstor F. Orlando-Indacochea ${ }^{\mathrm{I}}$

nestor.orlando@unesum.edu.ec

$$
\begin{aligned}
& \begin{array}{l}
\text { Miguel A. Osejos-Merino II } \\
\text { miguel.osejos@unesum.edu.ec }
\end{array} \\
& \begin{array}{l}
\text { Julio J. Jaramillo-Véliz } \\
\text { julio.jaramillo@unesum.edu.ec }
\end{array}
\end{aligned}
$$

Medardo A. Saltos-Bury IV

medardosaltosbury@hotmail.com

José L. Alcívar-Cobeña V

jose.alcivar@unesum.edu.ec

Correspondencia: nestor.orlando@unesum.edu.ec

\footnotetext{
I. Docente Universidad Estatal del Sur de Manabí, Jipijapa, Ecuador.

II. Docente Universidad Estatal del Sur de Manabí, Jipijapa, Ecuador

III. Docente Universidad Estatal del Sur de Manabí, Jipijapa, Ecuador.

IV. Docente Universidad Estatal del Sur de Manabí, Jipijapa, Ecuador.

v. Docente Universidad Estatal del Sur de Manabí, Jipijapa, Ecuador.
} 
Néstor F. Orlando-Indacochea, Miguel A. Osejos-Merino, Julio J. Jaramillo-Véliz, Medardo A. Saltos-Bury, José L. Alcívar-Cobeña

\section{Resumen}

Esta investigación titulada: "Prevalencia de Toxocara canis y su incidencia zoonótica ambiental en niños de la ciudad de Jipijapa". La metodología utilizada fue la investigación no experimental, descriptiva de tipo transversal, para determinar el cálculo del tamaño de la muestra (n), tuvo como base la ecuación para muestreo. Se escogieron las ciudadelas Eloy Alfaro, 3 de Mayo, Alberto Heredia y los barrios, La Gloria y El Progreso, con la finalidad de determinar la prevalencia de Toxocara canis en perros y niños. Una vez identificados los barrios y ciudadelas, se tomaron las muestras de heces de los perros en estudio, para procesarlas y analizarlas, utilizando el método de flotación con solución salina saturada. La muestra de los niños se escogió de aquellos que viven en casa con perros positivos a Toxocara canis, se utilizó la técnica de ELISA para la determinación inmunoenzimática cualitativa de anticuerpos específicos contra Toxocara canis, con una sensibilidad que varía entre el 80 y $100 \%$ y una especificidad del 90 - 95\%. Los resultados revelaron una prevalencia de Toxocara canis en canis domésticos del 22.7\% (75/330). La relación zoonótica de niños que conviven con canis domésticos positivos a toxócara canis es del 36\% (18/50). La prevalencia y la relación zoonótica de perros y niños es bastante alta, por lo tanto se crea la necesidad de ampliar la forma diagnostica y laboratorial de la toxocariosis humanas y en perros.

Palabras claves: Toxocara canis, prevalencia, zoonótica, ambiental, Jipijapa. 
Prevalencia de Toxocara canis y su incidencia zoonótica ambiental en niños de la ciudad de Jipijapa

\section{Abstract}

This research entitled: "Prevalence of Toxocara canis and its environmental zoonotic incidence in children of the city of Jipijapa.". The methodology used was non-experimental, descriptive crosssectional investigation, to determine the calculation of the sample size (n), based on the equation for sampling. The cities of Eloy Alfaro, 3 de Mayo, Alberto Heredia and the neighborhoods, La Gloria and El Progreso, were chosen in order to determine the prevalence of Toxocara canis in dogs and children. Once the neighborhoods and citadels were identified, stool samples were taken from the dogs under study, to be processed and analyzed, using the flotation method with saturated saline solution. The sample of the children was chosen from those who live at home with dogs positive to Toxocara canis, the ELISA technique was used for the qualitative immunoenzymatic determination of specific antibodies against Toxocara canis, with a sensitivity that varies between 80 and $100 \%$ and a specificity of $90-95 \%$. The results revealed a prevalence of Toxocara canis in domestic canis of $22.7 \%$ (75/330). The zoonotic relationship of children who live with positive domestic canis to toxócara canis is $36 \%(18 / 50)$. The prevalence and zoonotic relationship of dogs and children is quite high, thus creating the need to expand the diagnostic and laboratory form of human toxocariosis and in dogs

Key words: Toxocara canis, prevalence, zoonotic, environmental, Jipijapa. 
Néstor F. Orlando-Indacochea, Miguel A. Osejos-Merino, Julio J. Jaramillo-Véliz, Medardo A. Saltos-Bury, José L. Alcívar-Cobeña

\section{Introducción.}

El ser humano como ente de la naturaleza y su relación con la misma, se habituó a convivir de manera armoniosa con la presencia de animales domesticados, en especial, perros y gatos, situación que comienza con la costumbre del hombre de acoger a determinados animales en su hogar, sin requerirles a cambio función alguna, exceptuando la de su propia compañía, apareciendo así las mascotas (acompañantes de los seres humanos en su vida cotidiana, que no son destinados al trabajo ni tampoco son sacrificados para que se conviertan en alimento). Esta relación ambiental de mutuo beneficio entre dos seres vivos (hombre-animal) diferentes en la mayoría de sus aspectos, se puede tornar delicada si no se hace el debido cuidado del animal por parte del hombre, quien, como ser vivo consciente de sus acciones y responsable de las consecuencias de las mismas, puede afectar de manera negativa tanto al animal domesticado como a el mismo. Las enfermedades parasitarias han producido a través de los tiempos más muertes y daño económico a la humanidad que todas las guerras juntas, los países con poco desarrollo socioeconómico son en los que las parasitosis se presentan con mayor frecuencia (Gómez, 2014).

La Toxocariosis causada por el parásito Toxocara canis es una de las más importantes enfermedades parasitarias de los caninos, su distribución geográfica es muy alta y es un problema latente para la salud pública. Es una zoonosis parasitaria de transmisión directa a través del suelo o vegetales contaminados. Por lo que el propietario de un perro debe conocer cuáles son los parásitos que pueden afectar a su mascota, los síntomas que producen en los animales infestados y la forma más efectiva para el control y prevención en su caso particular.

La Toxocariosis puede ser adquirida de forma accidental, directa o indirecta de alimentos contaminados con huevos infectivos que producen en el hombre el síndrome conocido como larva migrante visceral caracterizada por lesiones granulomatosas crónicas asociadas a la presencia de parásitos en los órganos internos como hígado, pulmones, cerebro y ojos. En el perro el Toxocara canis clínicamente se caracteriza por disturbios entéricos provocados por el estado adulto y alteraciones viscerales en el hígado y pulmón. (Rivera Zambrano, 2014). 
Prevalencia de Toxocara canis y su incidencia zoonótica ambiental en niños de la ciudad de Jipijapa

Toxocara canis es un nematodo de los caninos, miembro de la familia Ascarididae, que accidentalmente infesta al hombre. Los cachorros expulsan los huevos con las heces a partir de la cuarta semana de vida. Con relación a la infestación humana, la mayoría de los trabajos realizados en nuestro país apuntan a determinar el grado de contaminación de los suelos. (García L. , López, Bojanich, Laffont, \& Alonso, 2012).

En la población canina, la Toxocariosis origina dificultades nutricionales, mala absorción de nutrientes, obstrucción intestinal, produciendo incluso la muerte del animal. Los órganos más afectados por la larva migrans visceral son hígado, pulmón y el sistema nervioso central. (Rivera Zambrano, 2014).

La infección se adquiere por contacto con los huevos fértiles larvados del parásito, que pueden persistir como infectantes hasta años, en suelo húmedo y temperatura templada; también, soportan la desecación por su cubierta muy resistente. Se describe las características y mecanismos de transmisión a los seres humanos, la distribución geográfica y, manifestaciones clínicas, resaltando su importancia como causa infecciosa de ceguera en individuos jóvenes, que puede ser potencialmente curable y prevenible por medio de la detección temprana y difusión de información en la población, para evitar la infección. La metodología de trabajo incluye la toma de muestras de los caninos y niños que conviven con perros infestados con toxocara. (Guarín, Situación de la Toxocariasis en algunos países de Latinoamérica: Revisión sistemática, 2014)

El diagnóstico se lo llevó a efecto en los laboratorios de la Universidad Estatal Del Sur De Manabí, que consiste en realizar un examen coprológico en los caninos, el diagnostico serológico se ejecutó mediante la demostración de anticuerpos específicos por medio de la prueba de ELISA en niños, en los laboratorios de International Laboratories Services INTERLAB S.A. El presente trabajo se lo realizó con la finalidad de impulsar la prevención a través de la detección precoz, promoviendo el cumplimiento sobre el cuidado responsable de mascotas, niños y la prevención de la contaminación del ambiente. 
Néstor F. Orlando-Indacochea, Miguel A. Osejos-Merino, Julio J. Jaramillo-Véliz, Medardo A. Saltos-Bury, José L. Alcívar-Cobeña

\section{Marco Teorico}

\subsection{Toxocara canis}

Toxocara canis es un nematode de los caninos, miembro de la familia Ascarididae, que accidentalmente infesta al hombre. Los cachorros expulsan los huevos con las heces a partir de la cuarta semana de vida. Con relación a la infestación humana, la mayoría de los trabajos realizados en nuestro país apuntan a determinar el grado de contaminación de los suelos. (García L., López, Bojanich, Laffont, \& Alonso, 2012)

\subsubsection{Clasificación taxonómica de Toxocara canis}

\section{Reino: Metazoa}

Subreino: Eumetazoa

Rama: Bilateria

Grado: Pseudocoelomata

Phylum: Nematoda

Clase: Chromadorea

Orden: Ascaridida

Superfamilia: Ascaridoidea

Familia: Toxocaridae

Género: Toxocara

Especie: canis. (Andrango M., Morales G., \& Jumbo M. 2013)

\subsubsection{Morfología}

Estos nematodos ascáridos son gusanos dioicos (hembra y macho). En la región anterior presentan una boca provista con tres labios bien desarrollados y alulas (aletas) cervicales y la vulva 
Prevalencia de Toxocara canis y su incidencia zoonótica ambiental en niños de la ciudad de Jipijapa

de la hembra. En la región media se aprecia el intestino y en la posterior las gónadas y la cloaca y papilas caudales de los machos. En promedio, las hembras miden unos $10-12 \mathrm{~cm}$ de longitud y los machos 4 - $6 \mathrm{~cm}$. Otras características diagnósticas del género son la ornamentación de la cutícula y las espículas desiguales. Los huevos son esféricos, color marrón oscuro, con cubierta externa gruesa e irregular y miden 75 a $90 \mu \mathrm{m}$. (Uribarren, 2015)

\subsubsection{Biología. Ciclo Biológico}

La infección se mantiene en los perros o gatos según la especie de Toxocara, el perro se infecta al comer huevos embrionados, la hembra infectada puede transmitir las larvas a la descendencia por vía congénita, los perros parasitados contaminan la tierra por eliminación de huevos con las materias fecales, es en donde se da el inicio del ciclo de vida en el hombre, que se da al ingerir huevos embrionados de T. canis o T. cati, mismos que liberan larvas en el intestino; estas larvas llegan a vía sanguínea y se localizan en las vísceras principalmente el hígado; por vía arterial pueden llegar al ojo, SNC, etc. donde dan lugar a granulomas de cuerpo extraño. Las larvas no se desarrollan a parásitos adultos en el hombre (Uribarren, 2015).

Cuando un perro ingiere un huevo de Toxocara canis, la larva aflora en el intestino delgado y pasa a través de la pared intestinal para llegar a los pulmones viajando en el torrente sanguíneo. Una vez en los pulmones alcanza un segundo nivel en su desarrollo y después, por un reflejo de expectoración/deglución, es enviada nuevamente hacia la zona intestinal donde terminará de madurar. Las "lombrices" adultas depositan sus huevos en el intestino y son expulsados a través de las heces, permitiendo que el ciclo vuelva a iniciarse en otro animal, gracias, entre otras cosas, a la gruesa película protectora que tienen dichos huevos y que les permite sobrevivir durante mucho tiempo en el ambiente. (Macpherson, 2013)

Es muy frecuente encontrar Toxocara Canis en los cachorros, que son infectados por su madre. Las larvas pasan a través de la leche e incluso de la placenta. Por eso es tan importante llevar a cabo un programa serio y fundamentado de desparasitación en los cachorros, empezando a la temprana edad de dos o tres semanas, antes de que las lombrices puedan empezar a minar el sistema inmunitario y el funcionamiento orgánico de los perritos. 
Néstor F. Orlando-Indacochea, Miguel A. Osejos-Merino, Julio J. Jaramillo-Véliz, Medardo A. Saltos-Bury, José L. Alcívar-Cobeña

Los perros adultos suelen infectarse al comer algún "receptor" de los huevos, que puede ser desde un pequeño animal (pájaro, ratón, etc...) hasta carne cruda infectada o la deyección de otro perro. Por eso es tan importante educar a los perros para que no coman nada que encuentren por la calle y, también, tratar de evitar que anden olisqueando (mucho menos comiendo) las cacas de otros perros, cuyo estado de salud ignoramos. La parasitosis por Toxocara es una zoonosis que puede afectar a las personas. Si un ser humano ingiere una gran cantidad de huevos, estos pueden desarrollarse en sus órganos internos y causar serios daños viscerales. Cuando la infestación es de un grado menor aparecen signos de malestares leves o moderados. En algunos casos, el Toxocara puede llegar a alcanzar los ojos, causando ceguera en muchos casos. (Macpherson, 2013)

Las infecciones por Toxocara canis se consideran más frecuentes que las debidas a T. cati, el cual tiene una distribución similar a la de T. canis y no debe ser ignorado. Asimismo, es necesario mantener en mente una gran cantidad de ascáridos de animales que pueden causar enfermedad en el ser humano. Se estima que la hembra elimina unos 200.000 huevos/día. Los huevos de T. canis son muy resistentes y pueden sobrevivir en el ambiente, bajo condiciones apropiadas, durante años. A temperaturas de $10-30^{\circ} \mathrm{C}$, en ambiente húmedo y oxigenado, al cabo de 2 - 6 semanas, se desarrollan huevos embrionados infectantes con larvas L3 en su interior, infectantes tanto para hospederos definitivos (canidos, félidos) y paraténicos (humano, ganado, roedores, entre otros). (Uribarren, 2015)

Tiene relevancia el hallazgo de concentraciones de huevos en diferentes estadios de desarrollo, viables, en el pelaje de perros, debido al contacto estrecho que suele presentarse con ellos, principalmente por parte de niños. Contribuyen a la dispersión de los huevos el viento, la lluvia, las moscas, cucarachas y lombrices, y pueden permanecer infectantes durante meses. (Uribarren, 2015)

\subsubsection{Agente Causal. Formas Parasitarias - Morfología}

En Toxocara canis se encuentran huevos, larvas y parásitos adultos, a continuación se detalla cada uno de ellos. 
Prevalencia de Toxocara canis y su incidencia zoonótica ambiental en niños de la ciudad de Jipijapa

1. HUEVOS: Son similares a los de áscaris humanos, pero un poco mayores de tamaño, redondeados y con cubierta externa más irregular, miden entre 90 micras de longitud por 75 micras de ancho. El huevo embrionado con larva L3 es la forma infectante.

2. LARVAS: Son las únicas formas del parasito que afectan al hombre, miden aproximadamente 400 micras de longitud.

3. ADULTOS: Tanto hembras como machos son similares a los de A. lumbricoides, pero de menor tamaño. Los machos miden de 5 a $10 \mathrm{~cm}$ de longitud, mientras que las hembras pueden alcanzar los $18 \mathrm{~cm}$. (Uribarren, 2015)

\subsubsection{Especies. Toxocara sp.}

En este género se destacan dos especies por su distribución y huéspedes finales: T. canis y T. cati. Se transmiten comúnmente al ingerirse pasivamente los huevos embrionados que se encuentran contaminando suelos, fómites y/o alimentos, incluso el pelo de cachorros; estos huevos poseen una capa externa acelular que les permite resistir las condiciones adversas del medio ambiente: temperaturas extremas, diferentes rangos de humedad y variaciones de nutrientes, $\mathrm{pH}$, minerales $\mathrm{y}$ textura de suelos. En este sentido, los huevos conservan mejor su viabilidad en suelos que retienen la humedad, que es limitante para la supervivencia de la larva, tales como los que tienen en su estructura mayor cantidad de arcilla que de arena. Asimismo, en los perros puede ocurrir la transmisión transplacentaria, oral y lactogénica. (Morales \& Esquivia, 2014).

\subsection{Toxocariosis o Zoonosis.}

Los síndromes clínicos de la toxocariasis son efecto de la migración de las larvas L3 por vía sanguínea a diferentes órganos, entre ellos hígado, cerebro, ojos, músculo. Esta migración puede resultar en un cuadro asintomático o una enfermedad con múltiples signos y síntomas; esto depende de los órganos invadidos, la duración de la migración, la intensidad de la infección, la edad y la respuesta inmune que presente el hospedero. Las larvas dejan huellas de la migración: hemorragia, necrosis, infiltrados inflamatorios. Las manifestaciones causadas por las larvas se atribuyen a la gran cantidad de productos de secreción/excreción que producen (lectinas, mucinas, enzimas, que interactúan con la respuesta inmune del hospedero y la modulan), y a la presencia de una cubierta rica en mucina, que la larva abandona cuando ésta es cubierta por anticuerpos y células y que da 
Néstor F. Orlando-Indacochea, Miguel A. Osejos-Merino, Julio J. Jaramillo-Véliz, Medardo A. Saltos-Bury, José L. Alcívar-Cobeña

lugar a una respuesta inflamatoria. (Maizels, 2013) y (Macpherson, 2013). En resumen, los síndromes clínicos debidos a la toxocariasis están relacionados con la migración larvaria y la respuesta inmune que provocan. (Uribarren, 2015).

La toxocariosis es una zoonosis menospreciada, causada por larvas de los nematodos del género Toxocara. Es una geohelmintiasis de animales de gran importancia en salud pública. En nuestros ambientes rurales y suburbanos, se considera que la principal especie patógena es: Toxocara canis, parásito de cánidos, entre ellos perros, zorros, lobos, coyotes; también debe contemplarse la distribución global de Toxocara cati, parásito de félidos, cuyo papel en la infección ha sido subestimado. Otros ascáridos pueden estar involucrados, entre ellos Toxascaris leonina, Baylisascariasis procyonis. (Macpherson, 2013). Citado por (Uribarren, 2015)

Es una parasitosis que afecta sobre todo a niños, que mantienen contacto estrecho con sus mascotas y/o juegan en cajas de arena y parques públicos, susceptibles de estar contaminados con heces fecales disueltas de perros y gatos. También son sujetos en riesgo las personas que ingieren carne cruda de diversos animales. Se identifican dos síndromes "clásicos": larva migrans visceral (LMV) y larva migrans ocular (LMO). Actualmente, se consideran también la toxocariasis común o encubierta y la neurotoxocariosis. (Macpherson, 2013). Citados por (Uribarren, 2015)

\subsubsection{Toxocara canis}

La infección con unos pocos gusanos no produce de ordinario síntomas en los perros adultos. Pero en caso de infecciones masivas (varios centenares) en el intestino puede darse apatía, inapetencia, pelo desgreñado o erizado, debilidad y susceptibilidad a otras enfermedades, oclusiones intestinales e incluso obstrucción de las vías biliares. Las consecuencias son diarrea o estreñimiento, vómitos, sangre en las heces, anemia, etc. Las larvas migratorios pueden dañar a los órganos más afectados como riñones, hígado, pulmones (tos y neumonía son posibles síntomas), o los ojos. (Junquera, 2015)

Todos estos daños pueden darse también en los cachorros, que a menudo muestran un característico vientre hinchado, y en los que estos trastornos afectan negativamente al desarrollo y al crecimiento. Debido a su gran talla, los adultos pueden obturar y perforar el intestino del cachorro. Si no se tratan a tiempo las infecciones de los cachorros con Toxocara canis pueden ser mortales. 
Prevalencia de Toxocara canis y su incidencia zoonótica ambiental en niños de la ciudad de Jipijapa

Los seres humanos pueden infectarse al ingerir huevos infectivos por contacto directo o indirecto con heces contaminadas de los perros, pero ocasionalmente también por huevos en el pelaje de los perros. Los seres humanos actúan de hospedadores intermediarios, es decir, las larvas no completan su desarrollo a gusanos adultos en el intestino humano. (Junquera, 2015)

Pero emigran (por ello se las denomina larva migrans) atravesando la pared intestinal hacia varios órganos, causando varios tipos de síndromes: larva migrans visceral, si afecta a diversos órganos internos (mayormente el hígado, los pulmones y la pared intestinal, a veces también el sistema nervioso, incluido el cerebro), y larva migrans ocular, si afecta a los ojos. Los niños corren un riesgo especial de infectarse, pues es más probable que ingieran huevos en entornos contaminados (jardines, parques, etc.). Las infecciones humanas con unos pocos gusanos son de ordinario benignas y se curan espontáneamente en unas pocas semanas. Pero infecciones oculares no tratadas pueden causar ceguera, e infecciones viscerales pueden ser incluso fatales en casos extremos. (Junquera, 2015)

\subsection{Incidencia zoonótica ambiental}

Según la Organización Mundial de la Salud (OMS, 2005), la toxocariasis se encuentra ampliamente distribuida a nivel mundial, siendo endémica en la mayor parte de los países de América, África y Asia. La prevalencia de esta infección es difícil de establecer por la dificultad de un diagnóstico certero y a que esta enfermedad no es de notificación obligatoria. El hombre es un hospedador accidental, siendo los niños los más afectados debido a sus hábitos de juegos en parques y patios de casa contaminados con huevos del parásito. (Delgado \& Rodríguez, 2009) citado por (Mosquera, Medina, Lares, Delgado, Martínez, \& Ferrer, 2014)

En este caso, no se da el desarrollo completo de T. canis, sólo sobrevive el estadio larvario. La infección sobreviene cuando el humano ingiere los huevos embrionados del parásito. Las larvas libres invaden la pared intestinal, entran en las vénulas mesentéricas o por los vasos linfáticos y son transportadas principalmente a hígado, pulmón, cerebro, globo ocular, musculatura etc. Las larvas son atacadas por una reacción celular del hospedador, de naturaleza granulomatosa. De este modo se produce una patología conocida como Síndrome Larva Migrans Visceral (SLMV) o Síndrome Larva 
Néstor F. Orlando-Indacochea, Miguel A. Osejos-Merino, Julio J. Jaramillo-Véliz, Medardo A. Saltos-Bury, José L. Alcívar-Cobeña

Migrans Oftálmica (SLMO) en el caso que afecte al globo ocular (Despommier, 2003), (Delgado \& Rodríguez, 2009) Y (Mosquera, Medina, Lares, Delgado, Martínez, \& Ferrer, 2014)

De igual manera, (Devera, Blanco, Hernández, \& Simoes, 2008) evaluaron 25 plazas y/o parques en Ciudad Bolívar y encontraron la presencia de huevos de Toxocara sp. tanto en tierra (55\% de las plazas) como en heces de perros (16,7\% de las plazas). Estos resultados muestran el riesgo potencial de transmisión de esta zoonosis en plazas y parques. El diagnóstico de la toxocariasis humana resulta difícil porque la sintomatología es poco específica, el parásito adulto no se encuentra en las heces ni tampoco sus huevos y el hallazgo de las larvas en los tejidos mediante biopsias de los granulomas es un hecho excepcional. La toxocariasis humana es una zoonosis parasitaria de amplia distribución y poco reconocida, aunque no se encuentra dentro de las enfermedades desatendidas, podría ser considerada como tal. Los antígenos que han demostrado mayor sensibilidad y especificidad son los antígenos de excreción/secreción del parásito (Delgado \& Rodríguez, 2009) citado por (Mosquer, Medina, Lares, Delgado, Martínez, \& Ferrer, 2014)

Entre las presentaciones clínicas, se han descrito cuatro síndromes de toxocariasis, conocidos como síndrome de toxocariasis encubierta o subclínica, larva migrans visceral (LMV), neurotoxocariasis y síndrome de larva migrans ocular (LMO). (Strube, Heuer, \& Janecek, 2013) y (Smith, Holland, Taylor, Magnaval, Schantz, \& Maizels, 2009). La presentación clínica clásica no patognomónica incluye eosinofilia elevada hasta 50\% o más, sola o acompañada de hepatomegalia, infiltrados pulmonares, tos y sibilancias, fiebre y linfadenopatía (LMV). En niños mayores o adolescentes, la migración o presencia de una sola larva en ojo resulta en una inflamación granulomatosa conducente a diversas manifestaciones oculares (LMO). (Smith, Holland, Taylor, Magnaval, Schantz, \& Maizels, 2009) y (Hotez \& Wilkins, 2009). Citado por (Mosquera, Medina, Lares, Delgado, Martínez, \& Ferrer, 2014)

\subsubsection{Importancia en salud pública}

La prevalencia de parasitosis intestinales en animales domésticos, especialmente perros y gatos y la dificultad de prevenir contaminación fecal al ambiente en ámbitos poco higiénicos con perros y gatos deambulando libres, representa un potencial importante de transmisión zoonótica al humano al contaminar el ambiente con huevos de Toxocara canis, Ancylostoma caninum o Echinococcus spp. o quistes y ooquistes de protozoos (Giardia spp o Cryptosporidium spp.) 
Prevalencia de Toxocara canis y su incidencia zoonótica ambiental en niños de la ciudad de Jipijapa

expulsados en las heces. Publicaciones recientes destacan el interés creciente de la infección de T. canis en el humano, conocida como toxocariasis humana, considerada como la más importante parasitosis desatendida en los Estados Unidos y de importancia a nivel global. (Strube, Heuer, \& Janecek, 2013) y (Hotez \& Wilkins, 2009). Citados por (Latorre \& Nápoles, 2014)

\section{Metodología}

\subsection{Ubicación Geográfica de la Investigación}

El presente trabajo de investigación se llevó a cabo en la ciudad de Jipijapa, localizado al extremo suroeste de la provincia de Manabí, entre las siguientes coordenadas:

Longitud Oeste $80^{\circ} 25^{`}$ y $80^{\circ} 52^{`}$

Latitud Sur $\quad 1^{\circ} 10^{\circ}$ y $1^{\circ} 47^{`}(25)$

Referente a la zona sur de la provincia de Manabí, está conformada por los cantones Jipijapa, Paján y Puerto López y 24 de Mayo. Ocupan 3.441,49 km2 de superficie, que corresponde al 15,65\% de la provincia de Manabí. Su economía se sustenta básicamente en la producción agropecuaria supeditada a las inclemencias o bondades de la naturaleza, en la explotación de la pesca artesanal y en el naciente impulso del turismo.

\subsection{Métodos de la Investigación}

\subsubsection{Modalidad y Tipo de Investigación}

La modalidad de investigación es descriptiva - transversal y el tipo de investigación es no experimental.

\subsubsection{Métodos}

El método que se utilizó fue el inductivo - deductivo. 
Néstor F. Orlando-Indacochea, Miguel A. Osejos-Merino, Julio J. Jaramillo-Véliz, Medardo A. Saltos-Bury, José L. Alcívar-Cobeña

El método inductivo se aplica la observación y la encuesta para la recolección y registro de la información de fuentes primarias.

El método deductivo permitió cumplir con los objetivos planteados.

\subsubsection{Estadística Descriptiva o Inferencial}

El método de investigación que se desarrolló fue el descriptivo transversal.

Se utilizó un diseño completamente al azar, estratificado. Las observaciones se obtuvieron de los perros y de los niños que habitan en las tres ciudadelas y los dos barrios respectivamente: Ciudadela Eloy Alfaro, Tres de Mayo, Alberto Heredia, Barrio El Progreso y Barrio La Gloria.

El análisis de los datos se realizó mediante tablas de contingencia, en la que se utilizó la prueba de Chi cuadrado; para el efecto se trabajó con el Programa Estadístico de manejo de datos (SPSS).

\subsubsection{Población y Muestra}

Universo y tamaño de la muestra.

La población canina de la Ciudad de Jipijapa de acuerdo a la última campaña de vacunación del 2014 fue de 7640 perros. Se tomó a consideración la población de perros de tres ciudadelas y dos barrios que es de 2374 perros. Para calcular el tamaño de la muestra se aplicó la fórmula estadística y se determinó que el tamaño de la muestra fue de 330 casos.

$$
\boldsymbol{n}=\frac{\boldsymbol{Z}^{2} * \boldsymbol{P} * \boldsymbol{Q} * \boldsymbol{N}}{(\boldsymbol{N}-\mathbf{1}) * \boldsymbol{E}^{2}+\boldsymbol{Z}^{2} *(\boldsymbol{P} * \boldsymbol{Q})}
$$

En donde:

$\mathrm{n}=$ Tamaño de la muestra.

$\mathrm{Z}=$ Valor $\mathrm{Z}$ curva normal (1.96) 
Prevalencia de Toxocara canis y su incidencia zoonótica ambiental en niños de la ciudad de Jipijapa

$\mathrm{P}=$ Probabilidad de éxito (0.50)

$\mathrm{Q}=$ Probabilidad de fracaso $(0.50)$

N = Población (2374)

$\mathrm{E}=$ Error muestra $(0.05)$

El tamaño de la muestra fue de: $n=330.65$

La muestra fue de 330 perros, la cual se dividió de acuerdo al porcentaje de perros que tenía cada ciudadela y barrio en estudio de la ciudad de Jipijapa, para recolectar las muestras de heces.

\subsubsection{Técnicas}

Encuesta: Se utilizó la encuesta como un proceso interrogativo relacionado con las variables, dirigidas a los habitantes de las ciudadelas y barrios en estudios. Con el objetivo de conocer el sistema de vida que tienen los niños, perros, norma de higiene, tipos de alimentos que consumen los caninos y si tienen contactos directos los niños con los perros; como instrumentos de esta encuesta se utilizó el cuestionario desarrollado en base al objetivo propuesto de esta investigación.

\section{Método de diagnóstico.}

Examen copro-parasitario directo por método de flotación.

Se utilizó para detectar cualitativamente huevos de nemátodos, el principio de este método es hacer flotar elementos contenidos en las heces. Se utilizó una solución saturada de Cloruro de Sodio con densidad de 1:15 a 1:20. La densidad de las soluciones es modificada por la temperatura ambiental.

\section{Materiales:}

Vasos de plástico

Coladeras de malla fina

Cucharas de plástico 
Néstor F. Orlando-Indacochea, Miguel A. Osejos-Merino, Julio J. Jaramillo-Véliz, Medardo A. Saltos-Bury, José L.

Cubre y portaobjetos

Centrífuga

Tubos de ensayo

Gradillas

Solución de $\mathrm{NaCl}$

Técnica en caninos:

Se toma de cinco a diez gramos de heces de diferentes partes de la muestra.

Se agrega solución salina hasta obtener un volumen de $100 \mathrm{ml}$, la suspensión fecal se tamiza utilizando una coladera de malla fina.

Se llenan las tres cuartas partes del tubo de ensayo.

Se coloca en la centrífuga durante tres minutos a $2000 \mathrm{rpm}$.

Después de la centrifugación se obtienen dos o tres gotas del sobrenadante con una pipeta, y se colocan en el porta objetos.

Se observa al microscopio con el objetivo 10x.

La presencia de huevos no estima el grado de infección.

\section{Técnica en niños}

Se utilizó la técnica ELISA, un kit de diagnóstico para la determinación inmunoenzimática cualitativa de anticuerpos específicos contra Toxocara canis. Con una sensibilidad que varía entre el 80 y $100 \%$ y una especificidad de 90 al 95\%. Se seleccionó a los niños que conviven con caninos positivos a Toxocara Canis de la Ciudad de Jipijapa que pertenecen a las ciudadelas y barrios escogidos, se obtuvo una muestra de sangre de los niños (5cc) se la colocó en tubos de ensayo con un gel especial que proporcionó el laboratorio, luego se realizó el proceso de centrifugación a 3500 rpm por 15 minutos para la obtención del suero, las mismas son guardadas en refrigeración si pasan las 72 horas hay que congelarlas $\left(-70 . .-20^{\circ} \mathrm{c}\right)$. Las muestras de suero fueron procesadas mediante la técnica de ELISA para detectar antígenos específicos contra Toxocara. (Rivera Zambrano, 2014) 
Prevalencia de Toxocara canis y su incidencia zoonótica ambiental en niños de la ciudad de Jipijapa

\section{Resultados}

¿Cuál es el número de perros por hogar?

\begin{tabular}{|c|c|c|c|c|c|}
\hline \multicolumn{6}{|c|}{ Tabla $\mathbf{N}^{\circ} 1$} \\
\hline & & Frecuencia & Porcentaje & Porcentaje válido & $\begin{array}{l}\text { Porcentaje } \\
\text { acumulado }\end{array}$ \\
\hline \multirow[t]{6}{*}{ Válido } & Cuatro & 3 & 1,6 & 1,6 & 1,6 \\
\hline & Dos & 54 & 28,0 & 28,0 & 29,5 \\
\hline & Ocho & 1 & 0,5 &, 5 & 30,1 \\
\hline & Tres & 46 & 23,8 & 23,8 & 53,9 \\
\hline & Uno & 89 & 46,1 & 46,1 & 100,0 \\
\hline & Total & 193 & 100,0 & 100,0 & \\
\hline
\end{tabular}

Fuente: Habitantes de la ciudad de Jipijapa

Elaboración propia

\section{Grafico $\mathbf{N}^{\circ} \mathbf{1}$}


Néstor F. Orlando-Indacochea, Miguel A. Osejos-Merino, Julio J. Jaramillo-Véliz, Medardo A. Saltos-Bury, José L. Alcívar-Cobeña

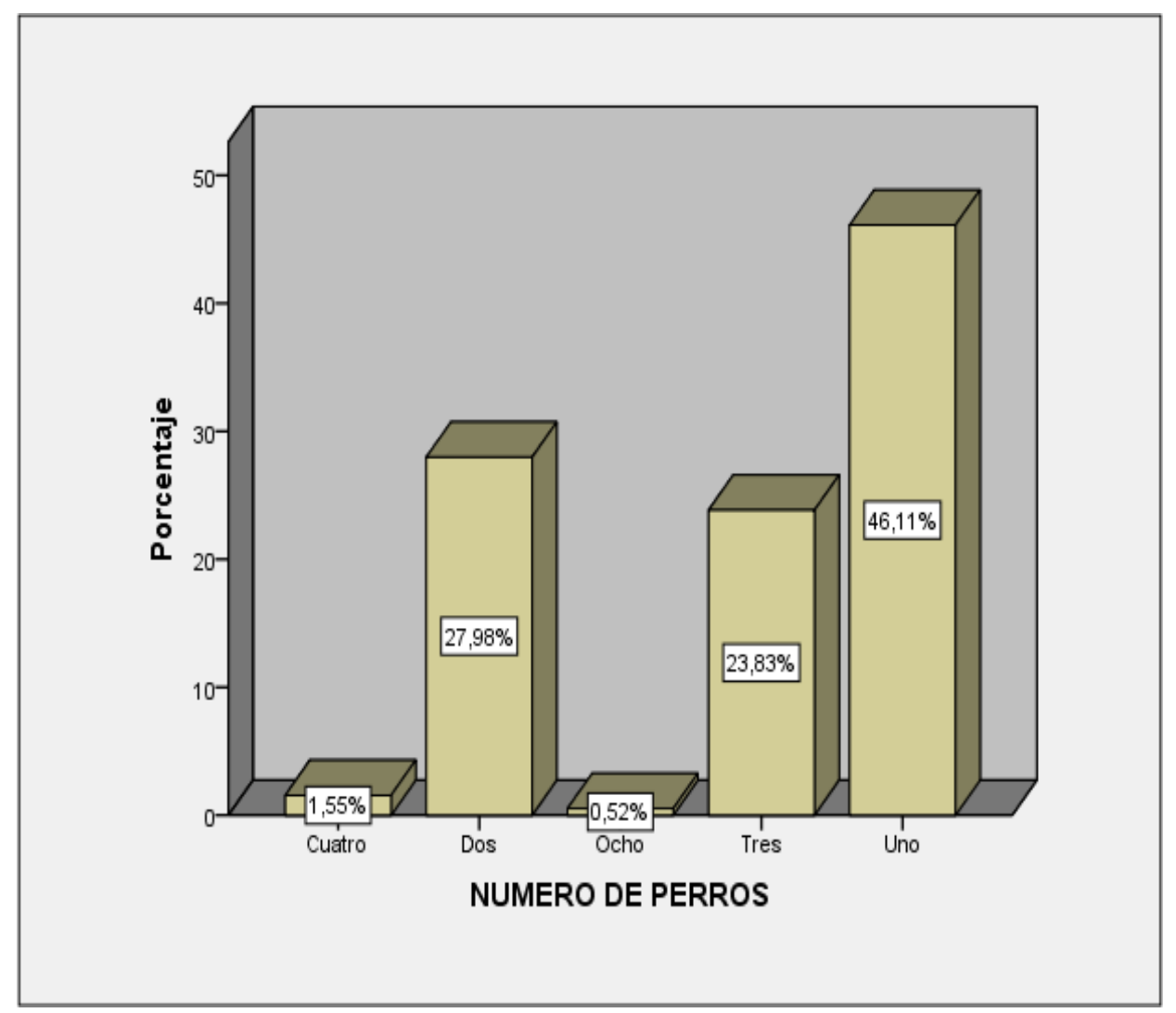

Fuente: Habitantes de la ciudad de Jipijapa

Elaboración propia

\section{Análisis e interpretación de resultados}

Analizado e interpretado los resultados según la tabla y gráfico $\mathrm{N}^{\circ} 1$ se pudo apreciar lo siguiente: el $46 \%$ manifestó que tienen un perro en su casa; el 28,7\% manifestó que tienen dos perros; el 23,8\% tiene hasta tres perros; el 1,5\% tiene hasta cuatro perros; finalmente el $0,5 \%$ que manifestó que tienen 8 perros en su hogar. En conclusión podemos mencionar que el mayor porcentaje representado por el $46 \%$ de la población en estudio vive con al menos un perro en su hogar.

\section{¿Usted desparasita a los perros?}

Tabla $\mathbf{N}^{\circ} 2$ 
Prevalencia de Toxocara canis y su incidencia zoonótica ambiental en niños de la ciudad de Jipijapa

\begin{tabular}{llllll}
\hline & Frecuencia & Porcentaje & $\begin{array}{l}\text { Porcentaje } \\
\text { válido }\end{array}$ & $\begin{array}{l}\text { Porcentaje } \\
\text { acumulado }\end{array}$ \\
\hline Válido & A Veces & 123 & 63,7 & 63,7 & 63,7 \\
& No & 41 & 21,2 & 21,2 & 85,0 \\
& Si & 29 & 15,0 & 15,0 & 100,0 \\
& Total & $\mathbf{1 9 3}$ & $\mathbf{1 0 0 , 0}$ & $\mathbf{1 0 0 , 0}$ & \\
\hline
\end{tabular}

Fuente: Habitantes de la ciudad de Jipijapa

Elaboración propia

Grafico $\mathbf{N}^{\circ} 2$ 
Néstor F. Orlando-Indacochea, Miguel A. Osejos-Merino, Julio J. Jaramillo-Véliz, Medardo A. Saltos-Bury, José L. Alcívar-Cobeña

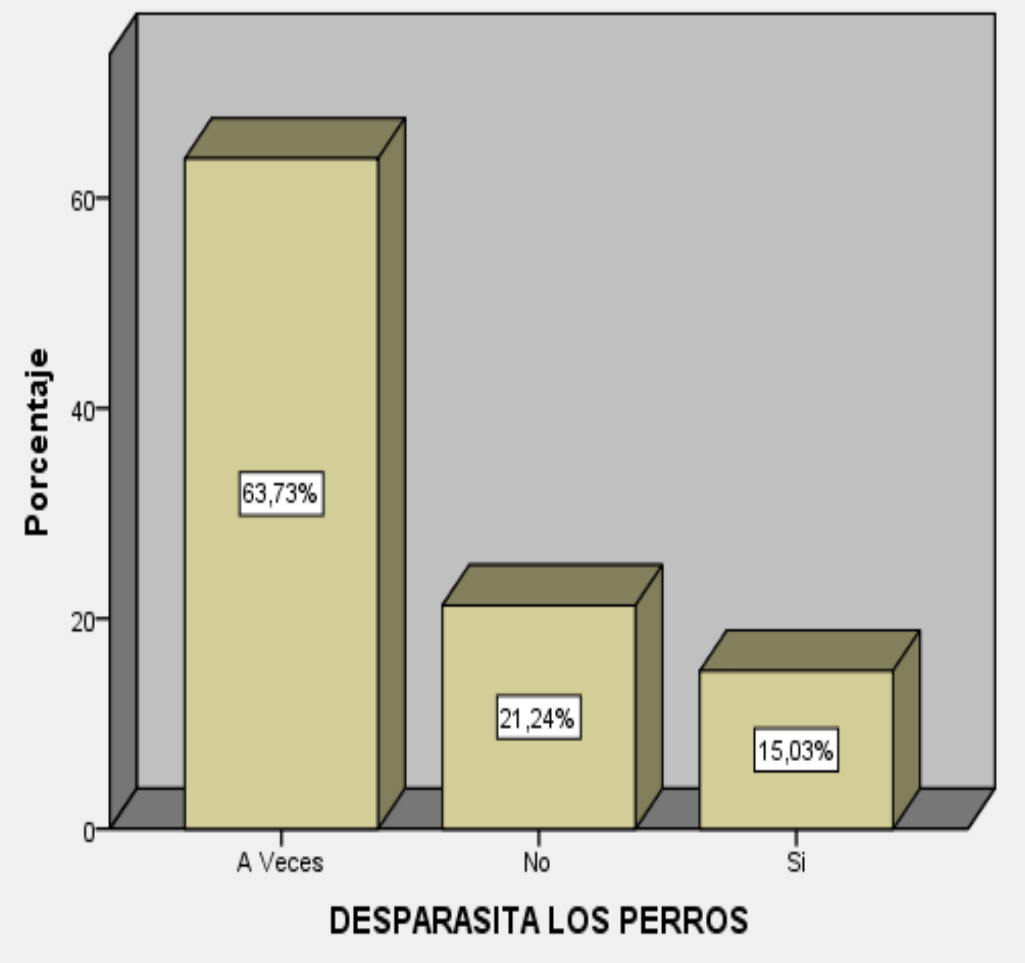

Fuente: Habitantes de la ciudad de Jipijapa

\section{Elaboración propia}

\section{Análisis e interpretación de resultados}

Una vez analizado e interpretado los resultados según la tabla y gráfico $\mathrm{N}^{\circ} 2$ se pudo apreciar que el 63,7\% manifestó que realizan una desparasitación esporádica (a veces), el 21,2\% manifestaron que no realizan desparasitación a sus perros, finalmente un $15 \%$ si realiza una desparasitación continua a sus perros. En conclusión podemos mencionar que el mayor porcentaje representado por el $63,7 \%$ de la población en estudio realiza una desparasitación esporádica (a veces). 
Prevalencia de Toxocara canis y su incidencia zoonótica ambiental en niños de la ciudad de Jipijapa

\section{¿Los niños tienen contacto con los perros?}

\section{Tabla $\mathbf{N}^{\circ} 3$}

\begin{tabular}{llllll}
\hline & & Frecuencia & Porcentaje & $\begin{array}{l}\text { Porcentaje } \\
\text { válido }\end{array}$ & $\begin{array}{l}\text { Porcentaje } \\
\text { acumulado }\end{array}$ \\
\hline \multirow{3}{*}{ Válidos } & No & 20 & 6.1 & 6.1 & 6.1 \\
& Si & 306 & 93.9 & 93.9 & 100.0 \\
& Total & $\mathbf{3 2 6}$ & $\mathbf{1 0 0 . 0}$ & $\mathbf{1 0 0 . 0}$ & \\
\hline
\end{tabular}

Fuente: Habitantes de la ciudad de Jipijapa

Elaboración propia

\section{Grafico $\mathbf{N}^{\circ} \mathbf{3}$}

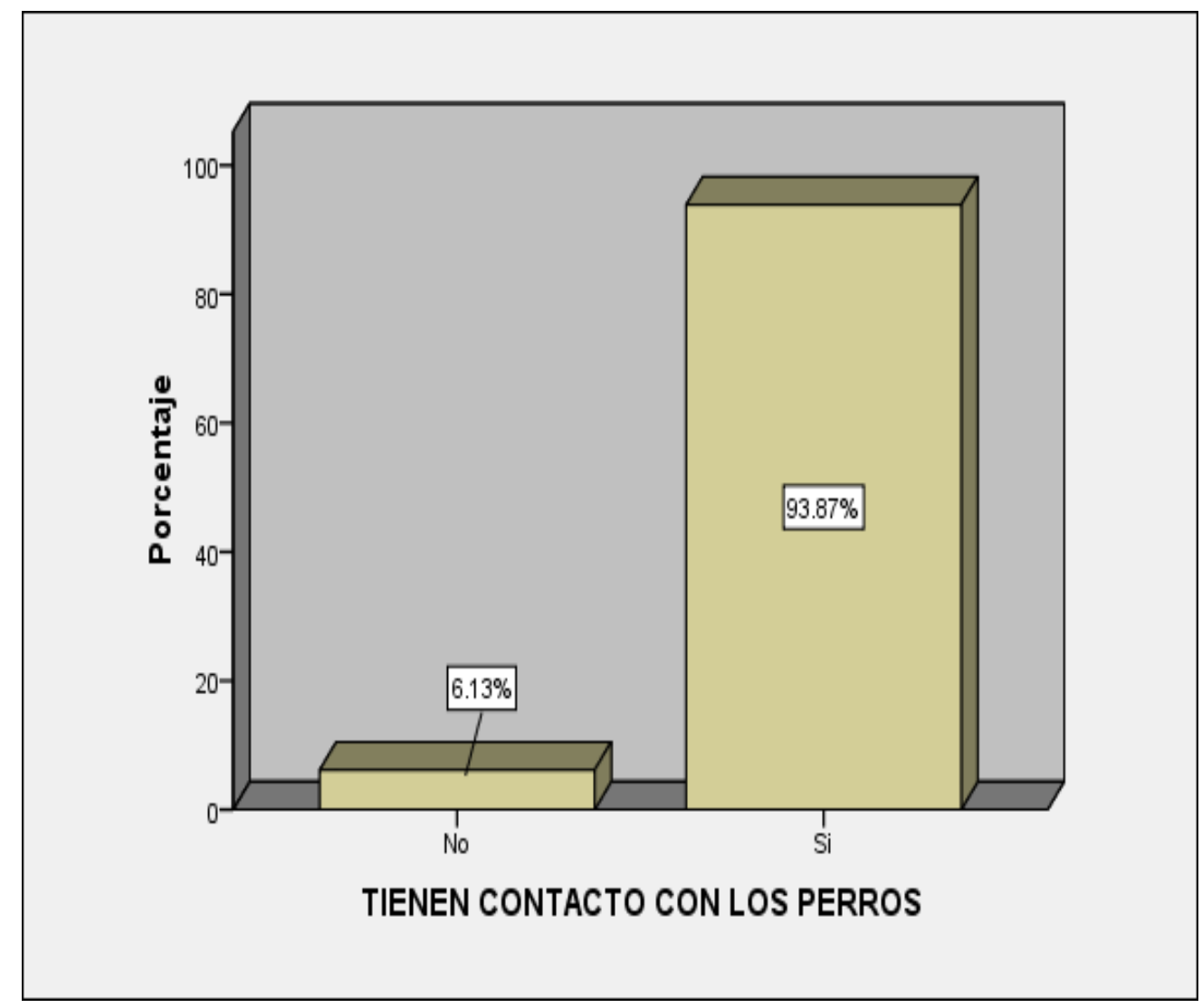

Fuente: Habitantes de la ciudad de Jipijapa Elaboración propia 
Néstor F. Orlando-Indacochea, Miguel A. Osejos-Merino, Julio J. Jaramillo-Véliz, Medardo A. Saltos-Bury, José L.

\section{Análisis e interpretación de resultados}

Analizado e interpretado los resultados según la tabla y gráfico $\mathrm{N}^{\circ} 3$ se pudo observar que el 93,87\% de los niños (as) si tienen contacto frecuente con los perros y el 6,13\% no tienen contacto con los canes. En conclusión podemos mencionar que el mayor porcentaje representado por el 93,87\% de los niños si tienen contacto con los perros.

¿Conoce usted el problema que ocasiona el toxocara canis en perros y niños?

Tabla $\mathbf{N}^{\circ} 4$

\begin{tabular}{llllll}
\hline & & Frecuencia & Porcentaje & $\begin{array}{l}\text { Porcentaje } \\
\text { válido }\end{array}$ & $\begin{array}{l}\text { Porcentaje } \\
\text { acumulado }\end{array}$ \\
\hline Válido & $\mathrm{Si}$ & 7 & 3,6 & 3,6 & 3,6 \\
& $\mathrm{No}$ & 186 & 96,4 & 96,4 & 100,0 \\
& Total & $\mathbf{1 9 3}$ & $\mathbf{1 0 0 , 0}$ & $\mathbf{1 0 0 , 0}$ & \\
\hline
\end{tabular}

Fuente: Habitantes de la ciudad de Jipijapa Elaboración propia 
Prevalencia de Toxocara canis y su incidencia zoonótica ambiental en niños de la ciudad de Jipijapa

\section{Grafico $\mathbf{N}^{\circ} 4$}

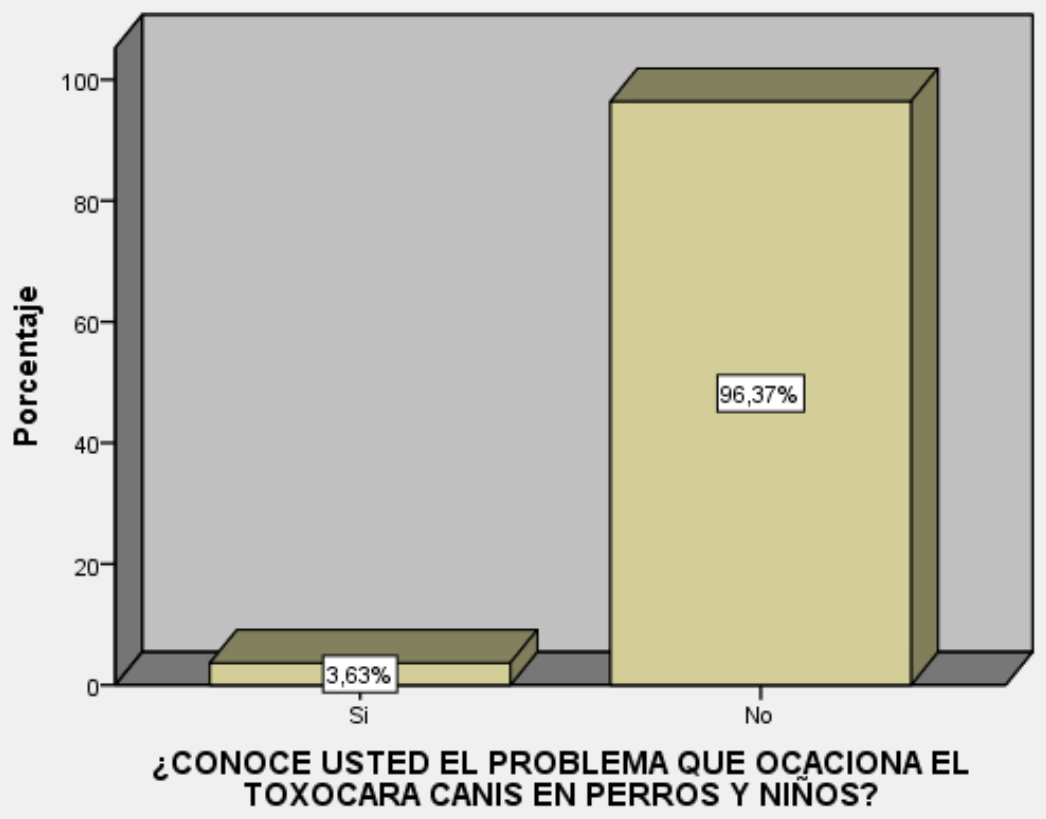

Fuente: Habitantes de la ciudad de Jipijapa

\section{Elaboración propia}

\section{Análisis e interpretación de resultados}

Una vez analizado e interpretado los resultados según la tabla y gráfico $\mathrm{N}^{\circ} 4$ se pudo observar que el 96,4\% manifestó que no tienen conocimiento referente al problema que ocasiona el Toxocara canis en perros y niños y un reducido 3,6\% si tiene conocimiento. En conclusión podemos mencionar que el mayor porcentaje representado por el $96,4 \%$ de los encuestados no tienen conocimiento referente al problema que ocasiona el Toxocara canis en perros y niños. 
Néstor F. Orlando-Indacochea, Miguel A. Osejos-Merino, Julio J. Jaramillo-Véliz, Medardo A. Saltos-Bury, José L. Alcívar-Cobeña

Determinación de la prevalencia de canis domésticos infestados con Toxocara canis

\section{Tabla $\mathbf{N}^{\circ} 5$}

Prevalencia de canis domésticos infestados con Toxocara canis

\begin{tabular}{cccc}
\hline & & Frecuencia & Porcentaje \\
\hline Válido & Positivo & 75 & 22,7 \\
& Negativo & 255 & 77,3 \\
& Total & $\mathbf{3 3 0}$ & $\mathbf{1 0 0 , 0}$ \\
\hline
\end{tabular}

Elaboración propia

Grafico $\mathbf{N}^{\circ} 5$

Determinación de la prevalencia de canis domésticos infestados con Toxocara canis

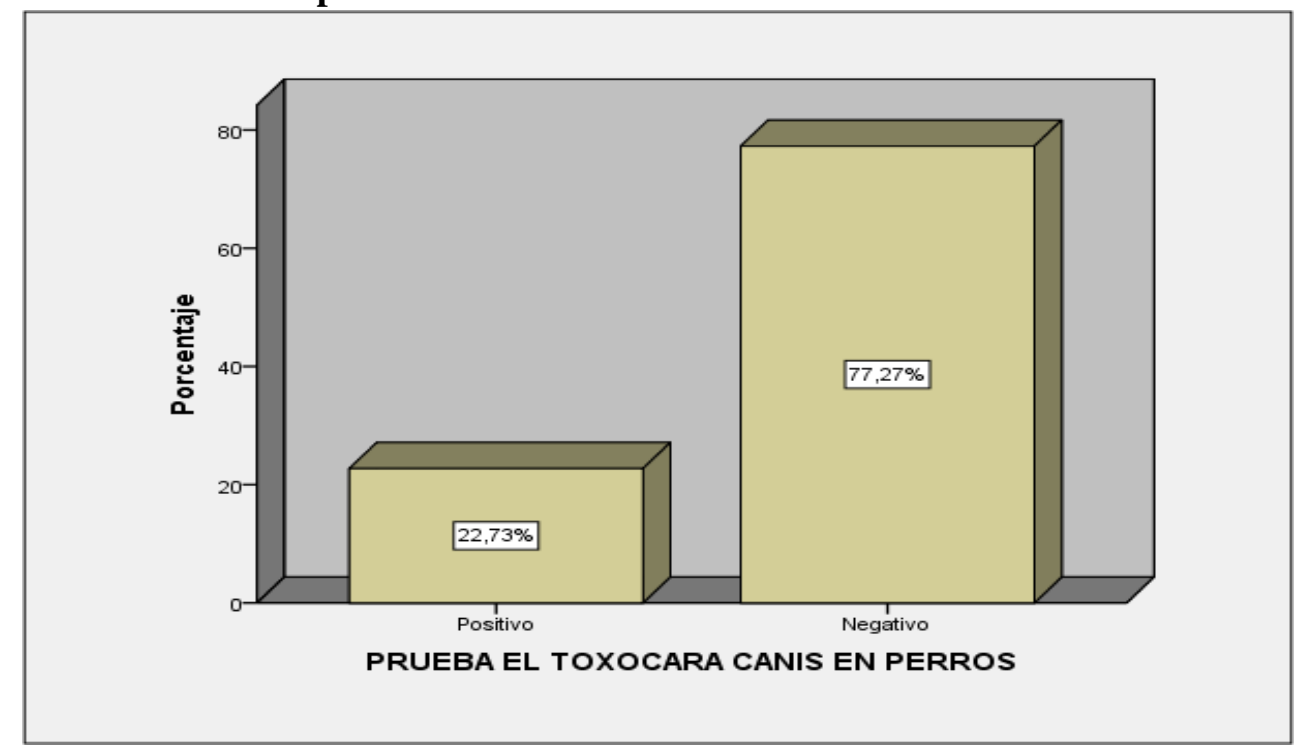

Elaboración propia

\section{Análisis e interpretación de resultados}

Analizado e interpretado los resultados según la tabla y gráfico $\mathrm{N}^{\circ} 5$ se pudo observar que se presenta la prevalencia de parásitos hallados en el estudio. De 330 caninos examinados, 255 $(77,27 \%)$ no presentaron Toxocara canis, en contraste con 75 perros $(22,73 \%)$ que dieron positivo 
Prevalencia de Toxocara canis y su incidencia zoonótica ambiental en niños de la ciudad de Jipijapa

de Toxocara canis. Los resultados de las pruebas de laboratorio demostraron que la prevalencia de Toxocara canis en los canes es del $22,73 \%$.

Casos de Toxocara canis en perros por sector

Tabla $\mathbf{N}^{\circ} 6$

Prevalencia de Toxocara canis en perros por sector

\begin{tabular}{|c|c|c|c|c|}
\hline \multirow[t]{2}{*}{ SECTOR } & & \multicolumn{2}{|c|}{$\begin{array}{l}\text { Prueba el Toxocara canis en } \\
\text { perros }\end{array}$} & \multirow[t]{2}{*}{ Total } \\
\hline & & Positivo & Negativo & \\
\hline & & 26 & 71 & 97 \\
\hline & 3 de Mayo & $26,80 \%$ & $73,20 \%$ & $100,00 \%$ \\
\hline & & 8 & 41 & 49 \\
\hline & Alberto Heredia & $16,30 \%$ & $83,70 \%$ & $100,00 \%$ \\
\hline & & 12 & 25 & 37 \\
\hline & El Progreso & $32,40 \%$ & $67,60 \%$ & $100,00 \%$ \\
\hline & & 17 & 73 & 90 \\
\hline & Eloy Altaro & $18,90 \%$ & $81,10 \%$ & $100,00 \%$ \\
\hline & & 11 & 46 & 57 \\
\hline & La Gioria & $19,30 \%$ & $80,70 \%$ & $100,00 \%$ \\
\hline \multirow{2}{*}{ Total } & & 74 & 256 & 330 \\
\hline & & $22,40 \%$ & $77,60 \%$ & $100,00 \%$ \\
\hline
\end{tabular}

\section{Elaboración propia}


Néstor F. Orlando-Indacochea, Miguel A. Osejos-Merino, Julio J. Jaramillo-Véliz, Medardo A. Saltos-Bury, José L. Alcívar-Cobeña

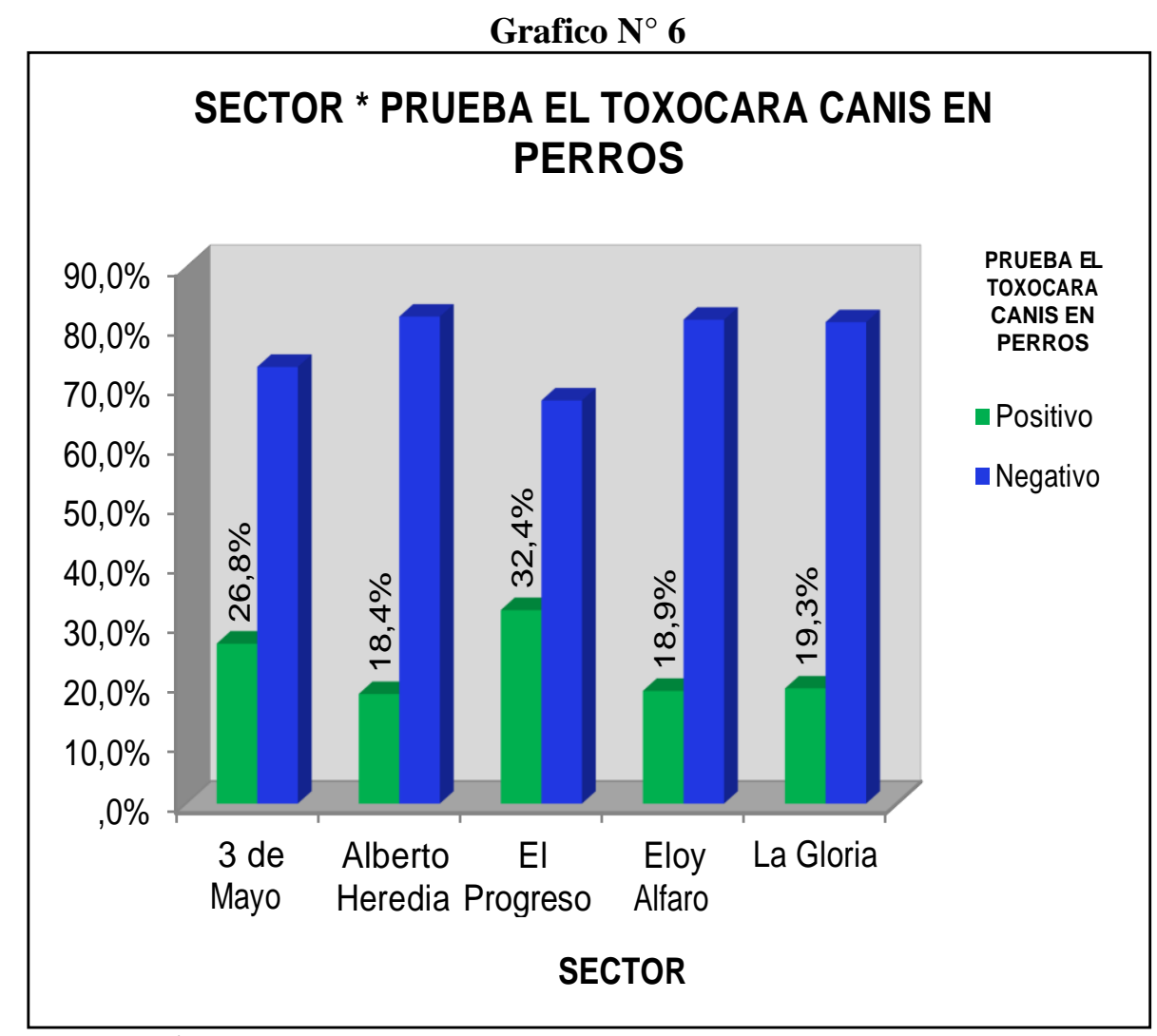

Elaboración propia

\section{Análisis e interpretación de resultados}

Una vez analizado e interpretado los resultados según la tabla y gráfico $\mathrm{N}^{\circ} 6$ se pudo apreciar que se presenta la prevalencia de parásitos hallados en el estudio. De 330 caninos examinados por sector, en relación a la ciudadela 3 de Mayo el 73,20\% no presentaron Toxocara canis y el 26,80\% fueron positivos, en el sector de Alberto Heredia el 83,70\% no presentaron Toxocara canis y el 16,30\% fueron positivos, en el sector del Progreso el 67,60\% no presentaron Toxocara canis y el 32,40\% fueron positivos, en el sector Eloy Alfaro el 81,10\% no presentaron Toxocara canis y el 18,90\% fueron positivos, finalmente en el sector de la gloria el 80,70\% no presentaron Toxocara canis y el 19,30\% fueron positivos. Los resultados de las pruebas de laboratorio demostraron que la mayor prevalencia de Toxocara canis en los canes se presentó en el sector El progreso con el $32,40 \%$. 
Prevalencia de Toxocara canis y su incidencia zoonótica ambiental en niños de la ciudad de Jipijapa

\section{Realización de un diagnóstico de laboratorio a niños que conviven con canis doméstico}

positivos para detectar la relación zoonótica.

\section{Tabla $\mathbf{N}^{\circ} 7$}

Relación zoonótica de niños que conviven con canis doméstico positivos

\begin{tabular}{llll}
\hline $\begin{array}{l}\text { Prueba de Elisa } \\
\text { en niños }\end{array}$ & Frecuencia & Porcentaje \\
\hline Válido & Negativo & 32 & 64,0 \\
& Positivo & 18 & 36,0 \\
& Total & $\mathbf{5 0}$ & $\mathbf{1 0 0 , 0}$ \\
\hline
\end{tabular}

Elaboración propia

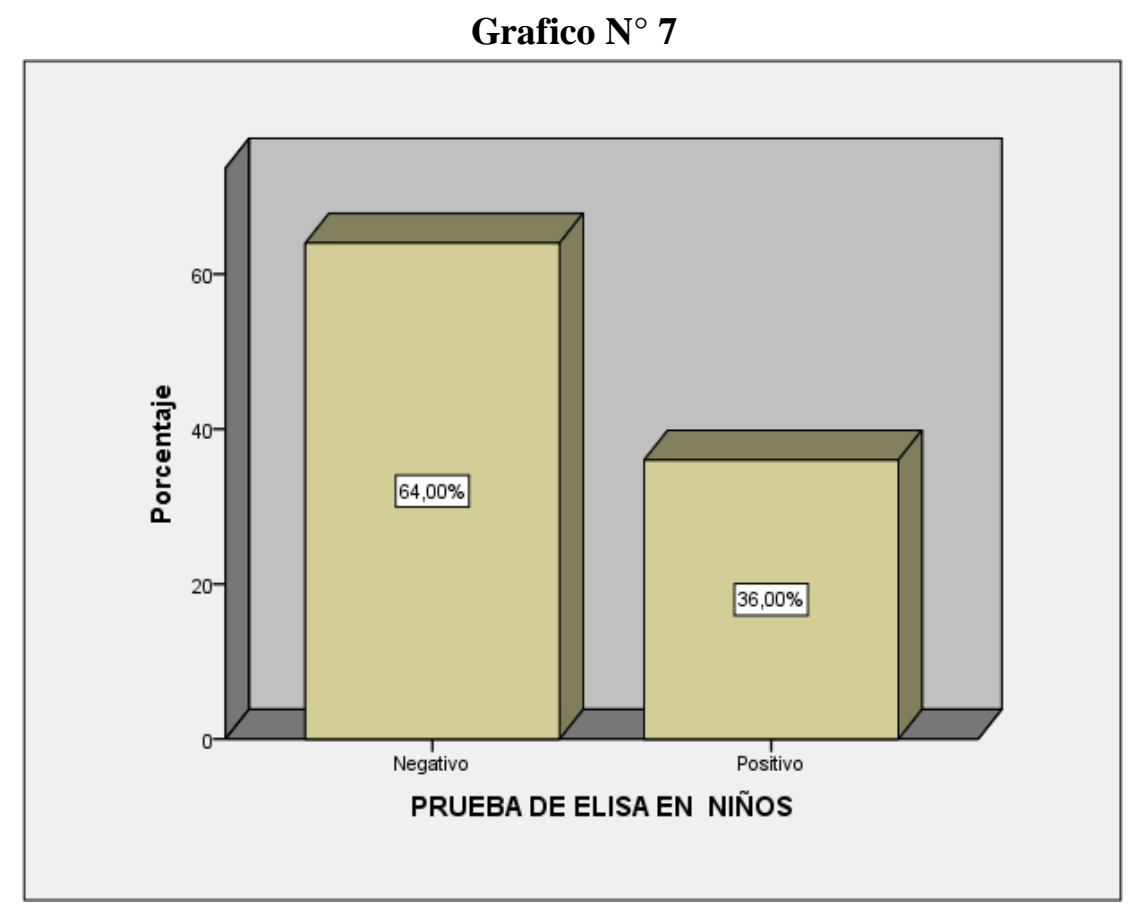

Elaboración propia

\section{Análisis e interpretación de resultados}

Analizado e interpretado los resultados según la tabla y gráfico $\mathrm{N}^{\circ} 7$ se pudo observar que de los 50 niños que se les realizó la prueba de Elisa, 32 niños (64\%) dieron negativo en la prueba de Elisa efectuada, en contraste con 18 niños (36\%) que dieron positivo en la prueba de Elisa 
Néstor F. Orlando-Indacochea, Miguel A. Osejos-Merino, Julio J. Jaramillo-Véliz, Medardo A. Saltos-Bury, José L. Alcívar-Cobeña

desarrollada. Los resultados de las pruebas de laboratorio demostraron que relación zoonótica de niños que conviven con canis doméstico positivos es del 36\%.

\section{Conclusiones}

- Los hallazgos ponen de manifiesto el papel de los perros con altas prevalencias de Toxocara canis $(22,7 \%)$, en la transmisión de esta enfermedad infecciosa zoonótica; los que por hábitos de defecación, en las calles, domicilios y plazas públicas, conducen, eventualmente, a la contaminación del medio ambiente, especialmente el suelo, el agua y la comida, poniendo en riesgo y amenaza la salud de la población, por la íntima relación de estos animales con el hombre, particularmente los niños.

- Se ha desarrollado la Prueba de ELISA para detección de Anticuerpos IgG anti- toxocara, de acuerdo al fabricante el referente de la prueba de ELISA tiene una sensibilidad que varía entre el 80 y $100 \%$ y una especificidad de 90-95\%, debido a que la relación zoonótica entre niños y canis domésticos positivos a Toxocara canis es alta (36\%), por ser la única técnica para detectar este parásito en humanos, en zonas tropicales que son endémicas de otras helmintiasis y poliparasitismo , las reacciones cruzadas suelen ser frecuentes, por lo tanto se obtienen falsos positivos en los resultados.

- Se debe crear conciencia en la población sobre la necesidad de la implementación de planes sanitarios adecuados y periódicos a las mascotas (perros y gatos), como desparasitación y vacunación, igualmente sobre la importancia de no llevar a estos animales a parques públicos donde 
Prevalencia de Toxocara canis y su incidencia zoonótica ambiental en niños de la ciudad de Jipijapa

concurren personas, esto con el fin de minimizar el riesgo de trasmisión de la Toxocariasis, cortando

el ciclo del parásito.

\section{Agradecimientos}

Agradecemos a las autoridades de la Universidad Estatal del Sur de Manabí (UNESUM), por el apoyo en esta investigación.

\section{Referencias Bibliográficas.}

Andrango M., Morales G., \& Jumbo M. (2013). Identificación de las especies de pulgas y endoparasitosis gastrointestinales asociadas en caninos en tres parroquias de la zona urbana (El Condado, San Juan y Quitumbe) del D.M.Q. (tesis de pregrado). Universidad Central del Ecuador, Quito, Ecuador.

Delgado, O., \& Rodríguez-Morales, A. (2009). Aspectos clínico-epidemiológicos de la toxocariasis: una enfermedad desatendida en Venezuela y América Latina. Boletín de Malariología y Salud Ambiental, 49(1):1-33.

Despommier, D. (2003). Toxocariasis: Clinical aspects, epidemiology, medical ecology and molecular aspects. Clinical Microbiology Reviews, 16(2): 265-272.

Devera, R., Blanco, Y., Hernández, H., \& Simoes, D. (2008). Toxocara y otros helmintos en plazas y parques de Cuidad Bolivar, Estado Bolivar, Venezuela. Enfermedades Infecciosas y Microbiología Clínica, 26(1): 23-26.

García, L., López, M., Bojanich, M., Laffont, H., \& Alonso, J. (2012). Detección de IgG anti Toxocara canis en perros de la Provincia de Corrientes, Argentina. Revista Veterinaria, 23(1): 69-70.

Guarín, C. (2014). Situación de la Toxocariasis en algunos países de Latinoamérica: Revisión sistemática (tesis de maestría). Universidad Nacional de Colombia. Facultad de Medicina, Instituto de Salud Pública, Departamento de Salud Pública. Bogotá, Colombia.

Hotez, P., \& Wilkins, P. (2009). Toxocariasis: America's most common neglected infection of poverty and a helminthiasis of global importance?. Plos Neglected Tropical Diseases, 3(3):14.

Junquera, P. (2015). TOXOCARA CANIS, gusano intestinal de los PERROS: biología, prevención y control. Parasitipedia.net

Latorre, E., \& Nápoles, M. (2014). Estudio Para Determinar la Contaminación con Parásitos Zoonóticos Caninos en Parques de la Zona Urbana del Distrito Metropolitano de Quito. 
Néstor F. Orlando-Indacochea, Miguel A. Osejos-Merino, Julio J. Jaramillo-Véliz, Medardo A. Saltos-Bury, José L. Alcívar-Cobeña

(tesis de pregrado).Universidad San Francisco de Quito. Colegio de Ciencias de la Salud. Quito, Ecuador.

Macpherson, CN. (2013). The epidemiology and public health importance of toxocariasis: A zoonosis of global importance. Int J Parasitol, 43(12-13): 999-1008.

Maizels, R. (2013). Toxocara canis: molecular basis of immune recognition and evasion. Veterinary Parasitology, 193(4):365-374., 365-374.

Morales, M., \& Esquivia, V. (2014). Contaminación de playas turisticas de la ciudad de cartagena de Indias con parásitos de importancia sanitaria 2012 - 2014. (tesis de maestria). Universidad de San Buenaventura. Fcaultad de Ciencias de la Salud. Cartegena de Indias.

Mosquera, S., Medina, O., Lares, M., Delgado, O., Martínez, M., \& Ferrer, E. (2014). Identificación de antígenos inmunodominantes específicos en productos de excreción/secreción de larvas de Toxocara canis. Saber, Universidad de Oriente, Venezuela. 26(3): 273-280.

OMS. (Abril de 2005). 14.a Reunión Interamericana a Nivel Ministerial en Salud y Agricultura. Organización Mundial de la Salud. Informe de la 4.a reunión de la comision

Panamericana de inocuidad de alimentos (COPAIA 4). Organización Mundial de la Salud OMS.México.

Organización Mundial de Sanidad Animal. (2013). Código Sanitario para los Animales Terrestres. Capítulo 7.7. Orfanizacióno Mundial de Sanidad Animal. Francia. Recuperado de http://www.oie.int/index.php?id=169\&L=2\&htmfile=chapitre_1.

Rojas, M. (2014). Toxocara canis en la Salud Pública Peruana. [Mensaje en un blog] Recuperado de http://mrojas.perulactea.com/2014/06/11/toxocara-canis-en-la-salud-publica-peruana-2/

Smith, H., Holland, C., Taylor, M., Magnaval, J., Schantz, P., \& Maizels, M. (2009). How common is human toxocariasis? Towards standardizing our knowledge. Trends Parasitol, 25(4): 1828.

Solarte, L., Castañeda, R., \& Pulido, A. (2012). Prevalencia de toxocara canis y ancylostoma caninum en materia fecal de caninos callejeros del centro de zoonosis de Bogotá. The Biologist, 10(2).

Strube, C., Heuer, L., \& Janecek, E. (2013). Toxocara spp. infections in paratenic hosts. Veterinary Parasitology, 193(4): 375-389.

Uribarren, T. (2015). Larva Migrans Visceral. México: Departamento de Microbiología y Parasitología, Facultad de Medicina, UNAM. Recuperado de http://www.facmed.unam.mx/deptos/microbiologia/parasitologia/larva-migrans-visceral.html 
Prevalencia de Toxocara canis y su incidencia zoonótica ambiental en niños de la ciudad de Jipijapa 